Provided for non-commercial research and education use. Not for reproduction, distribution or commercial use.

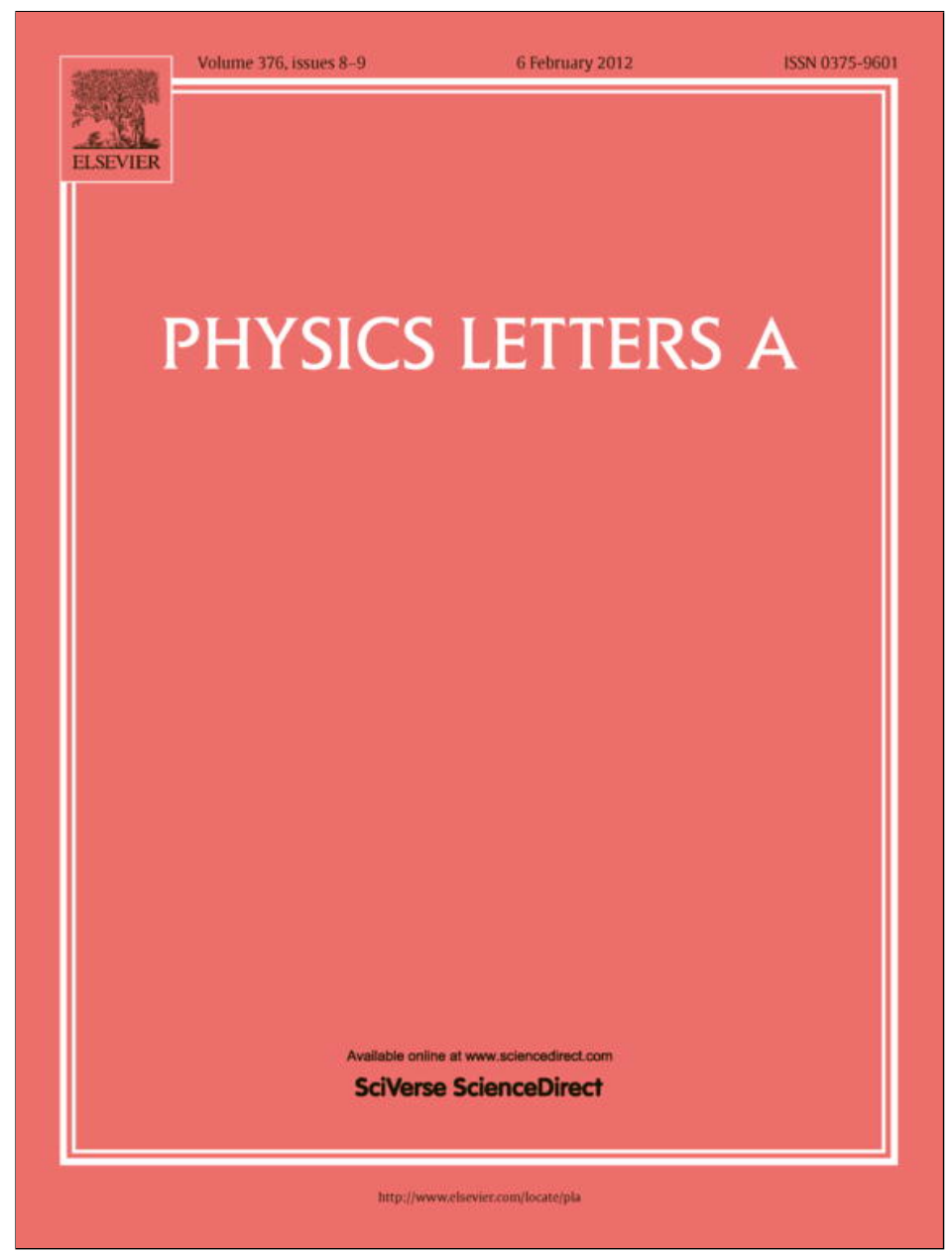

This article appeared in a journal published by Elsevier. The attached copy is furnished to the author for internal non-commercial research and education use, including for instruction at the authors institution and sharing with colleagues.

Other uses, including reproduction and distribution, or selling or licensing copies, or posting to personal, institutional or third party websites are prohibited.

In most cases authors are permitted to post their version of the article (e.g. in Word or Tex form) to their personal website or institutional repository. Authors requiring further information regarding Elsevier's archiving and manuscript policies are encouraged to visit:

http://www.elsevier.com/copyright 


\title{
Evolution of cubic-quintic complex Ginzburg-Landau erupting solitons under the effect of third-order dispersion and intrapulse Raman scattering
}

\author{
M.I. Carvalho ${ }^{a}$, M. Facão ${ }^{\text {b,* }}$ \\ a DEEC/FEUP and INESCPorto, University of Porto, Rua Dr. Roberto Frias, 4200-465 Porto, Portugal \\ b Department of Physics, I3N, University of Aveiro, Campus Universitário de Santiago, 3810-193 Aveiro, Portugal
}

\section{A R T I C L E I N F O}

\section{Article history:}

Received 6 August 2011

Received in revised form 29 December 2011

Accepted 24 January 2012

Available online 28 January 2012

Communicated by A.P. Fordy

\begin{abstract}
A B S T R A C T
The effects of third-order dispersion (TOD) and intrapulse Raman scattering (IRS) on the erupting solitons of the complex cubic-quintic Ginzburg-Landau equation are investigated by direct numerical simulations and linear stability analysis. Our results indicate that positive TOD eliminates eruptions on the leading edge of the soliton, whereas negative TOD cancels them on the other side. Moreover, the combined action of TOD and IRS is in certain cases able to eliminate explosions on both sides of the soliton, at much lower IRS values than with IRS alone. The profiles of the stationary solutions are increasingly asymmetric with TOD, and their velocity varies almost linearly with IRS.
\end{abstract}

(C) 2012 Published by Elsevier B.V.

\section{Introduction}

In the optics context, the complex cubic-quintic Ginzburg-Landau equation has been applied to pulse propagation in fibers with linear and nonlinear gain and spectral filtering [1-4] or pulse generation in fiber lasers with additive pulse mode-locking or nonlinear polarization rotation [5,6]. Among the large variety of solitary solutions it allows [7-13], all of them resulting form the balance between dispersion and nonlinearity, and between gain and loss, there are erupting solitons. As their name suggests, they propagate as a stationary pulse for a while, until they develop side eruptions that grow, and eventually break up and split in small humped waves that fade away. The single pulse survives this explosion and propagates steadily for another distance, with this process repeating itself with a period that is not exactly constant. The erupting solitons have been found numerically [12] on a relatively large region of the CGLE parameter space, and were experimentally observed in passively mode-locked lasers [14]. Recently, the eruptions were shown to change or even cancel by the introduction of one or more additional terms in the CGLE [15-20]. In fact, in [18,19] we have shown that the intrapulse Raman scattering (IRS), if sufficiently strong, may move one or both unstable eigenvalues to the stable region, thus eliminating the eruptions on one side, or on both sides, of the pulse. The observed steady pulses are slightly asymmetric and travel with a velocity that is proportional to the IRS strength. Moreover, it has been recently shown that it is possible to also obtain a fixed shape soliton from the combined action of IRS and third-order dispersion (TOD) $[17,20]$.

In this Letter, we consider the effects of TOD, acting alone or together with IRS, on the dynamics of erupting CGLE solitons. We focus our attention in two sets of the CGLE parameters on which the effect of both higher-order terms are significantly different. First, in Section 2, direct numerical simulations are used to investigate the evolution of these pulses, and to obtain their stationary profiles and velocity. Then, in Section 3, the observed dynamics are explained through a linear stability analysis which gives the location of both discrete and continuous eigenvalues, and respective eigenfunctions.

\section{Dynamics of erupting solitons with TOD and IRS}

The evolution equation considered here consists of the CGLE in the anomalous-dispersion regime plus two higher-order terms representing IRS and TOD,

$$
i q_{Z}+\frac{1}{2} q_{T T}+|q|^{2} q=i \delta q+i \beta q_{T T}+i \epsilon|q|^{2} q+i \mu|q|^{4} q-v|q|^{4} q+T_{r}\left(|q|^{2}\right)_{T} q+i \beta_{3} q_{T T T}
$$

\footnotetext{
* Corresponding author

E-mail addresses: mines@fe.up.pt (M.I. Carvalho), mfacao@ua.pt (M. Facão).
} 


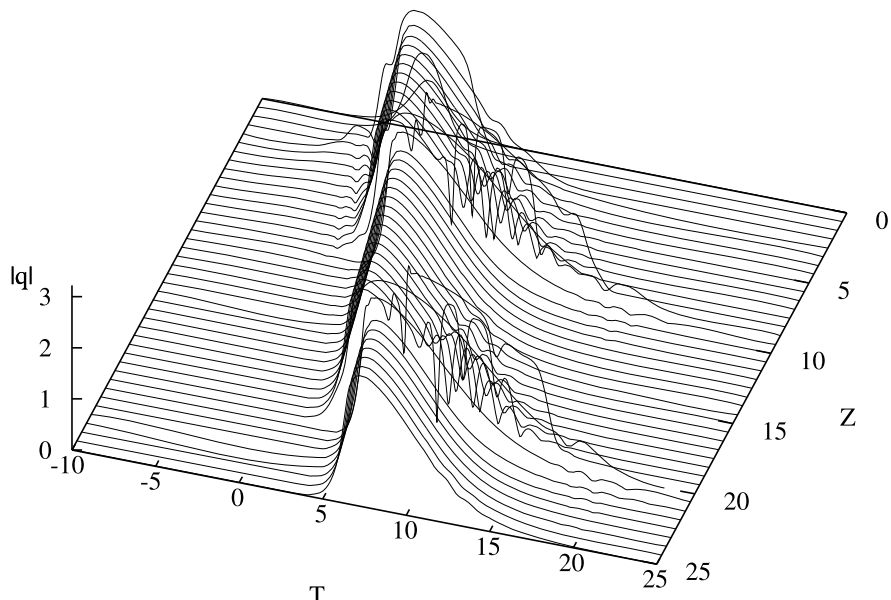

(a)

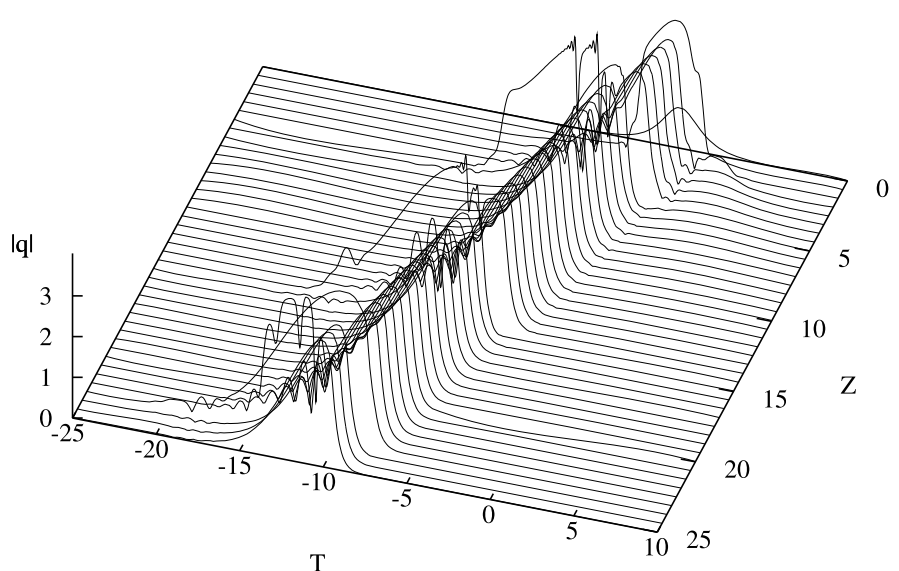

(b)

Fig. 1. Pulse evolution for (a) $\epsilon=1.0$ and $\beta_{3}=0.03$ and (b) $\epsilon=1.5$ and $\beta_{3}=-0.02$.

where, in the optical context, $q$ is the normalized envelope of the optical field and $Z$ and $T$ are the normalized propagation distance and retarded time, respectively. The parameters in this equation are all normalized versions of the actual parameters, namely, $\delta$ stands for linear gain/loss, $\epsilon$ for nonlinear gain, $\mu<0$ for the saturation of nonlinear gain, $\nu<0$ for the saturation of the Kerr effect and $T_{r}$ and $\beta_{3}$ are associated with IRS and TOD, respectively.

In this work, we are interested in a range of CGLE parameters for which erupting solitons have been found (see Fig. 5 of [12]), that is a region on the parameter plane $(\nu, \epsilon)$ for fixed $\beta=0.125, \delta=-0.1$ and $\mu=-0.1$. More specifically, we will consider here two sets of CGLE values inside this region, defined by the above parameters, $\nu=-0.6$, and $\epsilon=1.0$ in one case and $\epsilon=1.5$ in the other. Recently, we have shown that, on the above region, Eq. (1) with $\beta_{3}=0$ admits traveling single-hump solutions [18,19]. These solutions are of the form $q(Z, T)=F(\tau) e^{i \theta(\tau)+i \omega Z}$ ( $F$ and $\theta$ real), with $\tau=T-v Z$. The numerical simulations of Eq. (1) with $T_{r}=0$ but $\beta_{3} \neq 0$ suggest that also, in this case, there are traveling single-hump solutions. Unfortunately, there is no such a straightforward way to integrate the resulting ODE as the one previously used for the CGLE plus IRS. Hence, we proceed to study the eruption soliton dynamics using the PDE simulation results.

\subsection{PDE simulation results}

The effect of TOD over the erupting solitons, as far as we have observed for the two sets of CGLE parameters, may be summarized as the cancellation of the eruptions on the leading side of the soliton whenever $\beta_{3}>0$ and of the eruptions on the trailing side of the soliton otherwise (see Fig. 1). This symmetry breaking that is introduced by TOD has been referred earlier $[16,17,20]$. It is also interesting to mention that asymmetric explosions were reported for the CGLE without any higher-order term [21], but in that case the asymmetric explosions are not occurring on any preferred side of the pulse. In our simulations, we have observed that the dynamics for $\beta_{3}$ positive or negative are the same but just reversed from the trailing side to the leading side of the soliton. In effect, this result should have been expected since if $q(T, Z)=W(\tau) \exp (i \omega Z)$ with $\tau=T-v Z$ is solution of the CGLE plus positive TOD, then $q^{\prime}(T, Z)=W\left(\tau^{\prime}\right) \exp (i \omega Z)$ with $\tau^{\prime}=-T-v Z$ is the solution of the CGLE plus negative TOD. Note that this also gives that $\omega$ is the same, which corresponds to what has been observed. Furthermore, a certain threshold of TOD strength is necessary for the side eruptions to disappear. For instance, whether for $\epsilon=1.0,\left|\beta_{3}\right| \gtrsim 0.02$ is sufficient for canceling one side eruptions, for $\epsilon=1.5$, one needs $\left|\beta_{3}\right| \gtrsim 0.01$ to obtain the same effect. However, if $\beta_{3}$ is further increased it eventually reaches a value above which the eruption behavior is no longer present but instead we observe a chaotic kind of propagation. This change of behavior occurs around $\beta_{3}$ equal to 0.1 for $\epsilon=1.0$ and equal to 0.05 for $\epsilon=1.5$.

Before we proceed to the dynamics of the eruption solitons under the effect of both TOD and IRS, we will first remember the results obtained in $[18,19]$. For the CGLE parameters on the upper strip of the eruption region under study, there is always a threshold value of $T_{r}$ above which the eruptions are completely canceled and this threshold varies with $\epsilon$ and $\nu$. In fact, there is this main threshold for the complete suppression of eruptions but there is another critical $T_{r}$, below the main one, such that between them two there are only eruptions on one side of the soliton. Let us designate these critical $T_{r} \mathrm{~s}$ by $T_{r}^{\mathcal{C}_{1}}$ and $T_{r}^{\mathcal{C}_{2}}$ such that $T_{r}^{\mathcal{C}_{1}}<T_{r}^{\mathcal{C}_{2}}$. Another important aspect of the eruption behavior under the effect of IRS is that the one side cancellation is on the trailing or leading edge of soliton depending on the CGLE parameter set. And this was the main reason for us to choose here the two sets $(\epsilon, \nu)$ equal to $(1.0,-0.6)$ and $(1.5,-0.6)$, since in the first case the eruptions that persist in the interval $\left[T_{r}^{C_{1}}, T_{r}^{C_{2}}\right]$ are situated in the leading side of the pulse but in the latter case the persistent eruptions are on the trailing edge.

Therefore, we have checked for the possibility of positive TOD to cancel the persistent eruptions for $\epsilon=1.0$ and $T_{r}$ in $\left[T_{r}^{c_{1}}, T_{r}^{c_{2}}\right]$ and of negative TOD to cancel the persistent eruptions for $\epsilon=1.5$ and $T_{r}$ in between the correspondent critical $T_{r}$ values. In fact, this was the case for $\epsilon=1.0$ but not for $\epsilon=1.5$. Hence, for $\epsilon=1.0$ and $T_{r}$ between the two critical thresholds but not too small, positive TOD above a certain value is able to remove the eruptions on the leading edge of the pulse such that it makes stable propagation possible. However, for smaller values of $T_{r}$ but still in that range, the eruptions are completely removed but only up to a certain $\beta_{3}$, i.e., there is also a minimum $\beta_{3}$ necessary to remove the persistent eruptions as in any other case but there is also a maximum $\beta_{3}$ above which we observe eruptions on the trailing side, that is, the ones that have been already canceled by $T_{r}$. Fig. 2 shows this kind of behavior for $\epsilon=1.0$ and $T_{r}=0.26$. Our explanation for this effect is that TOD is changing considerably the pulse profile such that the IRS effect is no longer able to control the eruptions that it was controlling for $\beta_{3}=0$. This will be further discussed on the next sections. This same scenario occurs for $\epsilon=1.5$ 


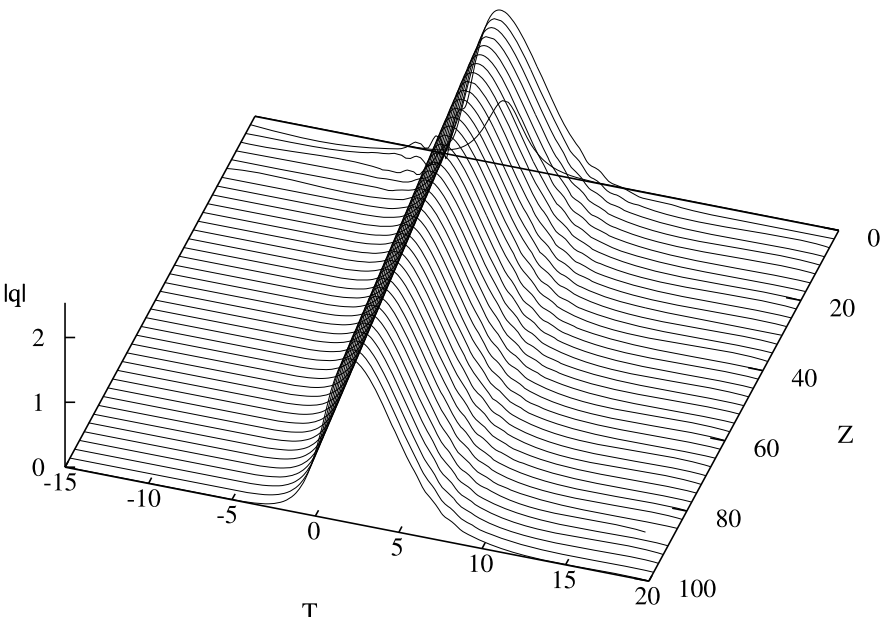

(a)

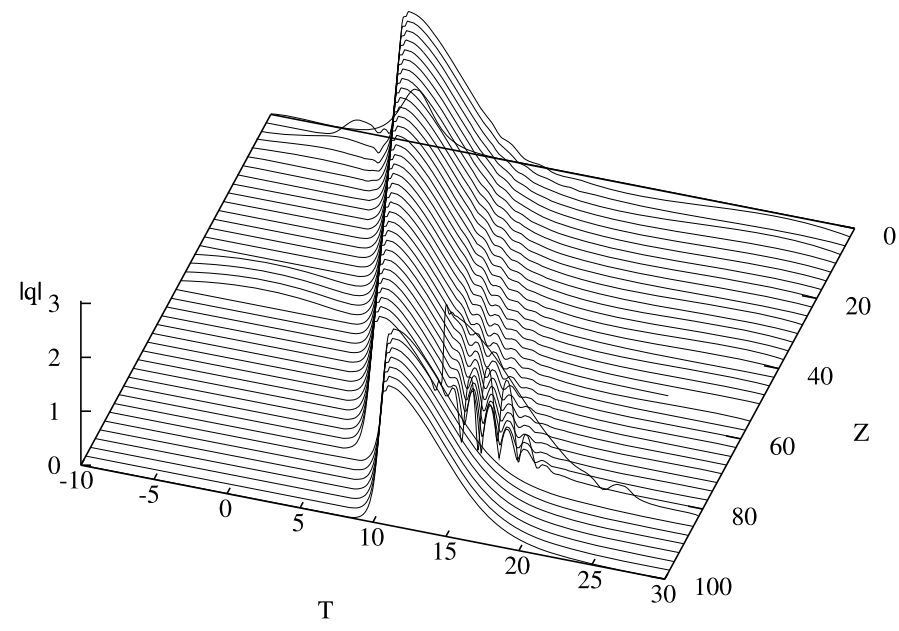

(b)

Fig. 2. Pulse evolution for $\epsilon=1.0, T_{r}=0.26$ and (a) $\beta_{3}=0.015$ and (b) $\beta_{3}=0.035$.

and every $T_{r}$ in the respective $\left[T_{r}^{c_{1}}, T_{r}^{c_{2}}\right]$, since whether the negative TOD get rid of the persistent trailing eruptions, the eruptions on the other side that are absent for $\beta_{3}=0$ will reappear for negative $\beta_{3}$ smaller than the value necessary to remove the trailing eruptions. Therefore, in this case we have not observed stable propagation for $T_{r}$ between the two thresholds and $\beta_{3}$ different from zero.

One different behavior that the introduction of IRS will bring is concerned with the upper limit of $\beta_{3}$ for pulse propagation. Although here we are mainly interested in pulse propagation in the eruption region, we report the observation of fronts for $\beta_{3}$ considerably larger than the limit for chaotic behavior referred above and $T_{r}$ in the interval defined by the two critical values.

\subsection{Steady profiles and velocity}

Our PDE simulations may also give us the steady profiles. Let us note that whether the simulations of the last subsection used a sech profile as input pulse in order to better excite the unstable modes and allow the eruptions to come up, if a pulse reasonably close to the steady one is used as input we observe a smooth adjustment to the actual steady pulse and the eruptions do not arise until very long $Z$ distances. These reasonably close pulse profiles were chosen to be the $\beta_{3}=0$ profiles that are obtained using a shooting method to integrate the corresponding ODE [18] or the output pulse from the PDE simulation for a closer $\beta_{3}$.

Let us first analyze the profiles for $T_{r}=0$ and $\beta_{3} \neq 0$. As mentioned above, in this case the steady profiles for symmetric $\beta_{3}$ are symmetric relatively to $T$ and have symmetric velocity. Hence, here we only present the results for positive $\beta_{3}$. As $\beta_{3}$ increases from zero, the pulse amplitude profile becomes asymmetric with a steeper edge on the leading side and a longer tail in the trailing side. Eventually, whenever $\beta_{3}$ is large enough, the profiles exhibit secondary peaks on the top of the main peak. Fig. 3 shows two sets of profiles for different values of $\beta_{3}$, Fig. 3(a) is for $\epsilon=1.0$ and Fig. 3(b) for $\epsilon=1.5$. There, it is notorious that the effect of TOD is stronger in the $\epsilon=1.5$ case, which can be explained by the fact that the stationary profiles are shorter in this case. The propagation constant $\omega$ first increases in modulus with $\beta_{3}$ but then decreases for the range of $\beta_{3}$ s that has profiles with secondary peaks. On the other hand, the velocities are positive for positive TOD and negative otherwise, and their absolute value increases with the absolute value of $\beta_{3}$. For both cases, $\epsilon=1.0$ and $\epsilon=1.5$, the velocity dependence with $\beta_{3}$ also changes whenever the profiles have secondary peaks on the top, namely, it increases at a larger rate.

The profiles and the $\omega$ values of the stationary pulses corresponding to the combined action of IRS and TOD are very similar to the ones obtained with $T_{r}=0$. This should have been anticipated since, as we have previously shown for $\beta_{3}=0$ [18], the major effect on the solution as $T_{r}$ varied was in the velocity $v$, which was found to be proportional to $T_{r}$ and negative. A similar result for the velocity is also obtained when considering that both IRS and TOD are present. In effect, as Fig. 4 shows for $\epsilon=1.0$, the velocity $v$ still decreases with $T_{r}$ in an approximately linear fashion. Moreover, the absolute value of the slope increases with $\beta_{3}$. Also note that since the value of the velocity for $T_{r}=0$ is positive for positive TOD, for each $\beta_{3} \neq 0$ there will be a fixed $T_{r}>0$ that corresponds to a stationary profile with zero velocity. For example, if we take the case $\beta_{3}=0.005$, such a pulse exists for $T_{r} \simeq 0.13$. Unfortunately, this value of $T_{r}$ is still bellow $T_{r}^{c_{1}}$, which means that, in this case, the IRS strength is not enough to eliminate the explosions on the trailing edge of the soliton, and therefore this pulse will exhibit the typical erupting behavior of this class of pulses. On the other hand, if we now consider $\beta_{3}=0.02$, we verify that the solution with $v=0$ will be propagating when $T_{r} \simeq 0.34$, that is, for a $T_{r}$ larger than $T_{r}^{c_{1}}$. Therefore, in this case the joint contribution of IRS and TOD is able to cancel explosions on both sides of the soliton, thus resulting in a pulse in the CGLE erupting region that propagates with no eruptions and with zero velocity.

\section{Linear stability analysis}

In order to better understand the different behavior of CGLE erupting solitons under the influence of IRS and TOD, we have investigated their linear stability spectrum. This spectrum can be obtained by assuming that the pulse consists of the above traveling solution plus a small perturbation term, namely, $q(Z, T)=\left[F(\tau) \mathrm{e}^{i \theta(\tau)}+\Delta(Z, \tau)\right] \mathrm{e}^{i \omega Z}$, and demanding that $\Delta$ has exponential dependence on $Z$, i.e., $\Delta(Z, \tau)=u(\tau) \mathrm{e}^{i \lambda Z}+x^{*}(\tau) \mathrm{e}^{-i \lambda^{*} Z}$, in which case we obtain the following stability eigenvalue problem

$$
L \mathbf{w}=\lambda \mathbf{w}, \quad \mathbf{w}=\left(\begin{array}{ll}
u & x
\end{array}\right)^{T}
$$




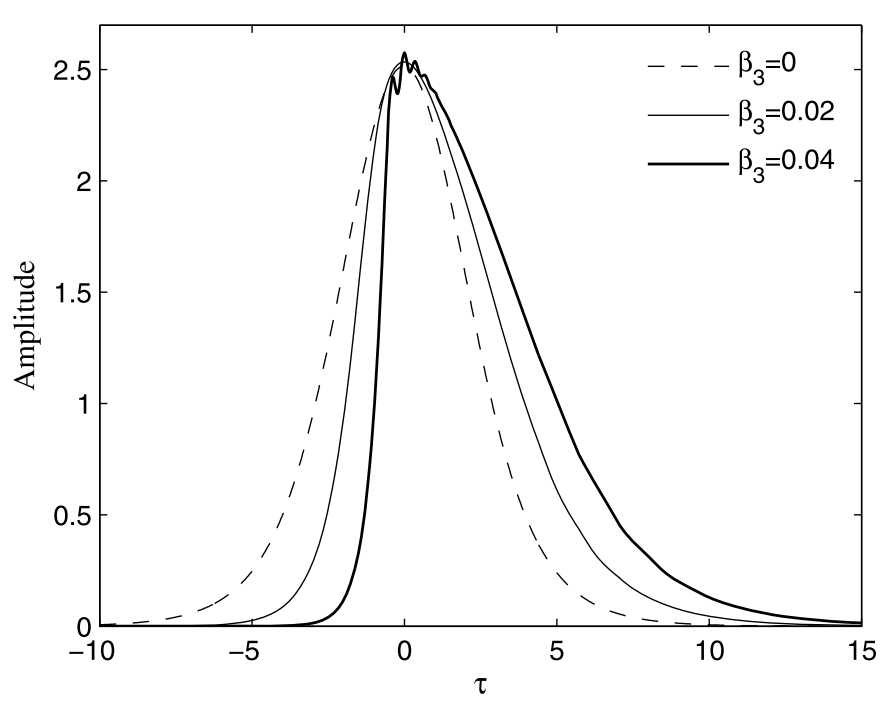

(a)

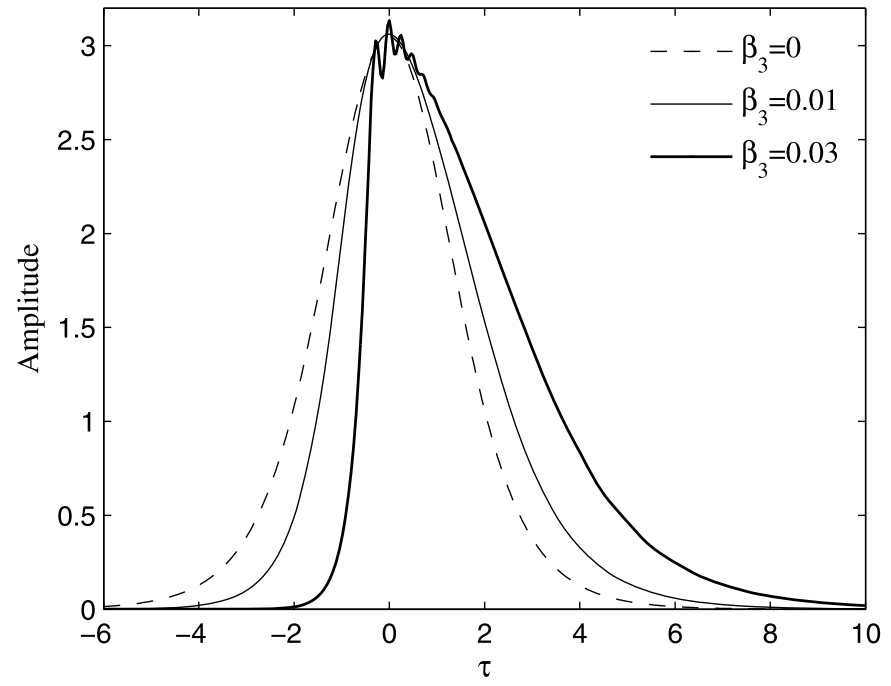

(b)

Fig. 3. Amplitude profiles for different values of $\beta_{3}$ and (a) $\epsilon=1.0$ and (b) $\epsilon=1.5$.

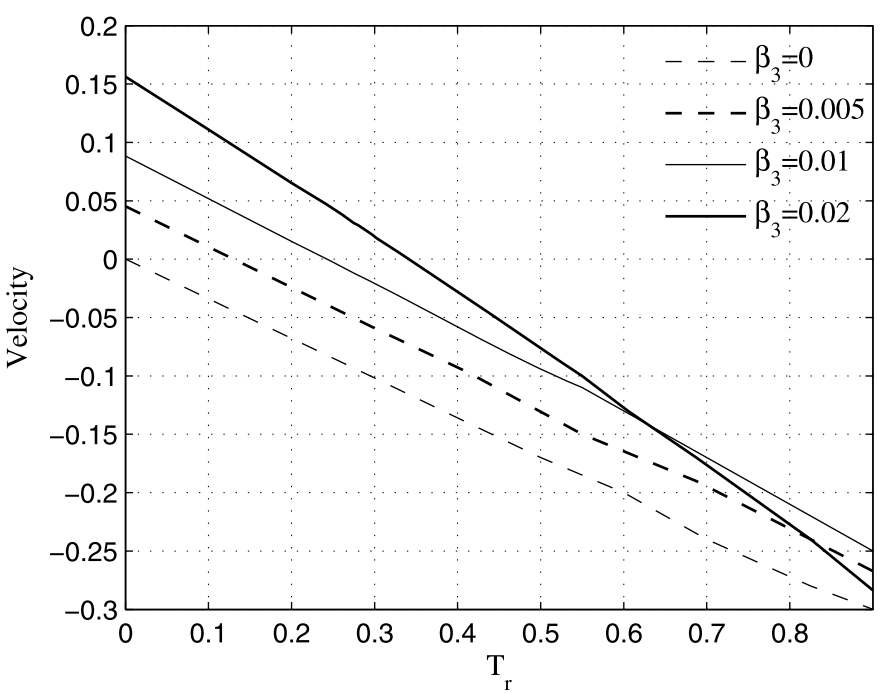

Fig. 4. Velocity of the stationary profiles for different values of $\beta_{3}$ and $T_{r}$, and for $\epsilon=1.0$.

where

$$
L=\left(\begin{array}{cc}
-i \beta_{3} \partial_{\tau \tau \tau}+\left(\frac{1}{2}-i \beta\right) \partial_{\tau \tau}-i v \partial_{\tau}-\omega-i \delta+2(1-i \epsilon) F^{2} & {\left[(1-i \epsilon) F^{2}-2(i \mu-v) F^{4}\right.} \\
-3(i \mu-v) F^{4}-T_{r}\left(3 F F^{\prime}+F^{2} \partial_{\tau}-i \theta^{\prime} F^{2}\right) & \left.-T_{r}\left(F F^{\prime}+F^{2} \partial_{\tau}+i \theta^{\prime} F^{2}\right)\right] \mathrm{e}^{2 i \theta} \\
{\left[-(1+i \epsilon) F^{2}+2(-i \mu-v) F^{4}\right.} & -i \beta_{3} \partial_{\tau \tau \tau}-\left(\frac{1}{2}+i \beta\right) \partial_{\tau \tau}-i v \partial_{\tau}+\omega-i \delta-2(1+i \epsilon) F^{2} \\
\left.+T_{r}\left(F F^{\prime}+F^{2} \partial_{\tau}-i \theta^{\prime}\right)\right] \mathrm{e}^{-2 i \theta} & +3(-i \mu-\nu) F^{4}+T_{r}\left(3 F F^{\prime}+F^{2} \partial_{\tau}+i \theta^{\prime} F^{2}\right)
\end{array}\right)
$$

The above expression for $\Delta$ implies that the traveling pulses are linearly stable if $L$ has no eigenvalues $\lambda$ with negative imaginary part. Note that, due to the symmetry of (2) relatively to the imaginary axis, whenever there is an eigenvalue $\lambda$, there also exists an eigenvalue $-\lambda^{*}$,

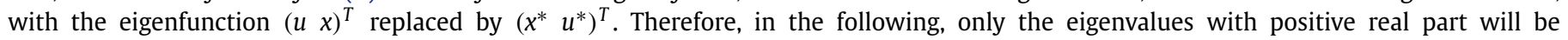
considered. The spectrum of $L$ consists of a continuous set and discrete eigenvalues. The location of the continuous spectrum may be estimated using the continuous spectrum of the limiting form of $L$ for $\tau \rightarrow \pm \infty$ which reads

$$
L_{\infty}=\left(\begin{array}{cc}
-i \beta_{3} \partial_{\tau \tau \tau}+\left(\frac{1}{2}-i \beta\right) \partial_{\tau \tau}-i v \partial_{\tau}-\omega-i \delta & 0 \\
0 & -i \beta_{3} \partial_{\tau \tau \tau}-\left(\frac{1}{2}+i \beta\right) \partial_{\tau \tau}-i v \partial_{\tau}+\omega-i \delta
\end{array}\right)
$$

The continuous spectrum of $L_{\infty}$ is composed by the curves on the complex plane $\left(\lambda_{r}, \lambda_{i}\right)$ defined by $\beta_{3} s^{3}+(1 / 2-i \beta) s^{2}-v s+\omega+$ $i \delta+\lambda=0$ and $\beta_{3} s^{3}-(1 / 2+i \beta) s^{2}-v s-\omega+i \delta+\lambda=0$, with $s$ being any real number. The existence of these two curves is once again associated with the symmetry of (2) with respect to the imaginary axis, and thus only the first one will be considered in the following. By substituting the new variables $z=\left(\lambda_{i}+\delta\right) / \beta$ and $t=\lambda_{r}+\omega+\left(\lambda_{i}+\delta\right) / 2 \beta$ in the equation above, it will take the simpler form $t^{2}=z\left(\beta_{3} z-v\right)^{2}$. The minimum value of $z$ is zero and it is attained at $t=0$, such that it corresponds to $\left(\lambda_{r}, \lambda_{i}\right)=(-\omega,-\delta)($ see Fig. 5). Note that a similar result was obtained in [19] for the CGLE in the presence of IRS. Since the continuous spectrum of $L$ itself is on the regions defined by the lines that constitute the continuous spectrum of $L_{\infty}$ [22], it is on or inside the above cubic curves. In turn, 


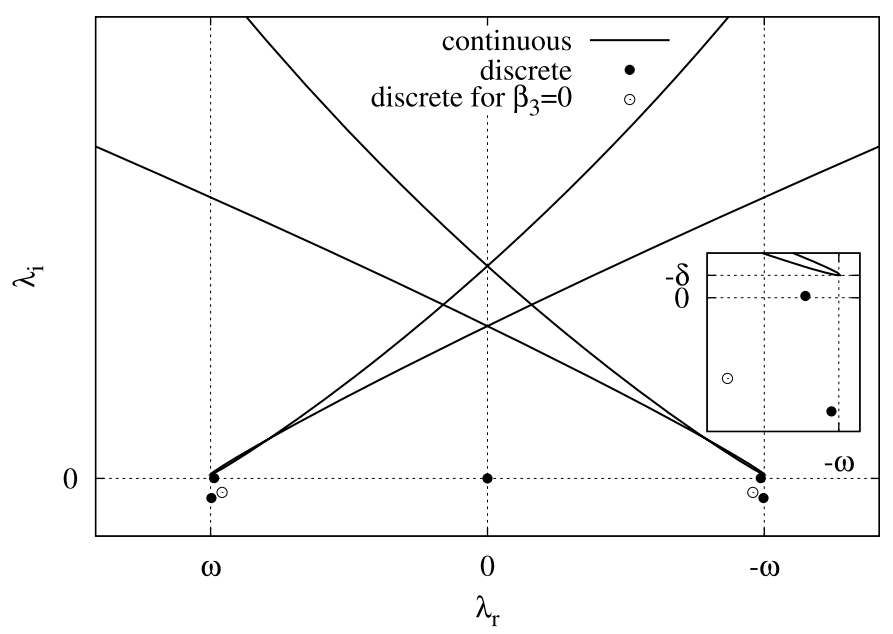

Fig. 5. Stability spectrum for $\delta=-0.1, \beta=0.125, \mu=-0.1, \epsilon=1.0, v=-0.6, T_{r}=0$ and $\beta_{3}=0.017$. The two lines are the boundary of the continuous spectrum and the black points are the discrete spectrum. The open points are the pair of double eigenvalues for the $\beta_{3}=0$ case. The inset shows the details of the lower part of the continuous and the discrete spectra.

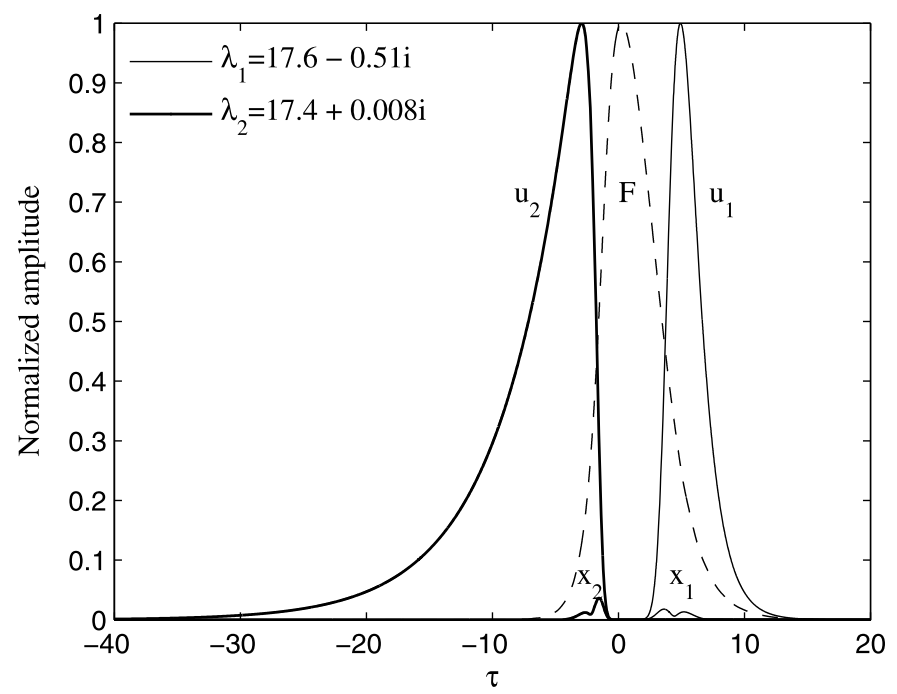

Fig. 6. Eigenfunctions corresponding to the discrete eigenvalues with positive real part for the previous values of $\delta, \beta, \mu$ and $\nu$, and for $\epsilon=1.0, T_{r}=0$ and $\beta_{3}=0.017$.

this implies that the continuous spectrum is located in the stable region of the $\lambda$-plane as long as $\delta<0$, that is, whenever there is loss to extinguish the linear waves.

As far as the discrete eigenvalues are concerned, there is one located at $\lambda=0$, which is double and corresponds to the translational and rotational invariances of the PDE (1). The other eigenvalues were computed by considering the discretized operator $L$, which is obtained by evaluating (2) in equidistant points and using finite-differences, and then applying sparse matrix methods. The continuous spectrum boundary, defined above, and the discrete eigenvalues are depicted in Fig. 5 for $\epsilon=1.0, T_{r}=0$ and $\beta_{3}=0.017$. As the inset on that figure shows, the double unstable eigenvalues for the $\beta_{3}=0$ case split up and travel by the effect of TOD, with one of them entering the stable region, and the other one being pushed further into the unstable half-plane. Fig. 6 shows the eigenfunctions associated with these eigenvalues. Note that both $u$ and $x$ are located to the right of the solitary pulse for the unstable eigenvalue, whereas the opposite can be observed for the other eigenvalue. Accordingly, it is expected that eruptions on the trailing edge of the solitary pulse are eliminated for the CGLE parameters considered here, $T_{r}=0$ and for this value of $\beta_{3}$, which we observed in our numerical simulations. Also note that the eigenfunctions of the stable eigenvalue are considerably larger than the other two, a result that can be easily explained by the closer proximity of this eigenvalue to the continuous spectrum.

Fig. 7 shows the trajectories of the discrete eigenvalues as $\beta_{3}$ varies for $\epsilon=1.0$, when no IRS is present (Fig. 7(a)) and under the combined action of TOD and IRS (Fig. 7(b)). In the first case, TOD makes both eigenvalues to move in separate directions, with one of them traveling toward the stable region while the other one goes deeper into the unstable one. Note that the real parts of both eigenvalues are modified by TOD in a similar fashion. It is also important to point out that for $\beta_{3}$ negative, the trajectories of both eigenvalues would be exactly the same as depicted in Fig. 7(a), with the only difference of swapping the leading and trailing edges. These results are in complete agreement with our numerical simulations, which show elimination of leading edge eruptions for $\beta_{3}$ positive, and trailing side ones otherwise. In order to better understand the elimination of eruptions on both sides of the soliton as a result of the combined effect of TOD and IRS, we have represented in Fig. 7(b) the trajectories for two values of $T_{r}, T_{r}=0.26$ and $T_{r}=0.3$, which are larger than $T_{r}^{c 1}$ (0.24) but smaller than $T_{r}^{c 2}(0.84)$. This means that in both cases the eigenvalue associated with the trailing edge eruptions is already stable when $\beta_{3}=0$, while the other one is not. This figure shows that the effect of positive $\beta_{3}$ on the leading edge eigenvalue when IRS 


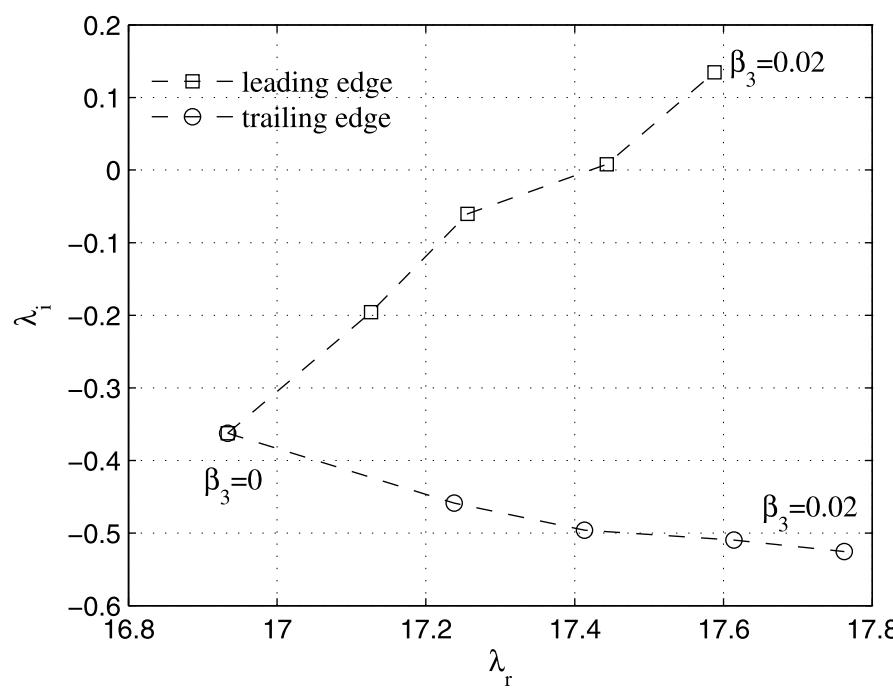

(a)

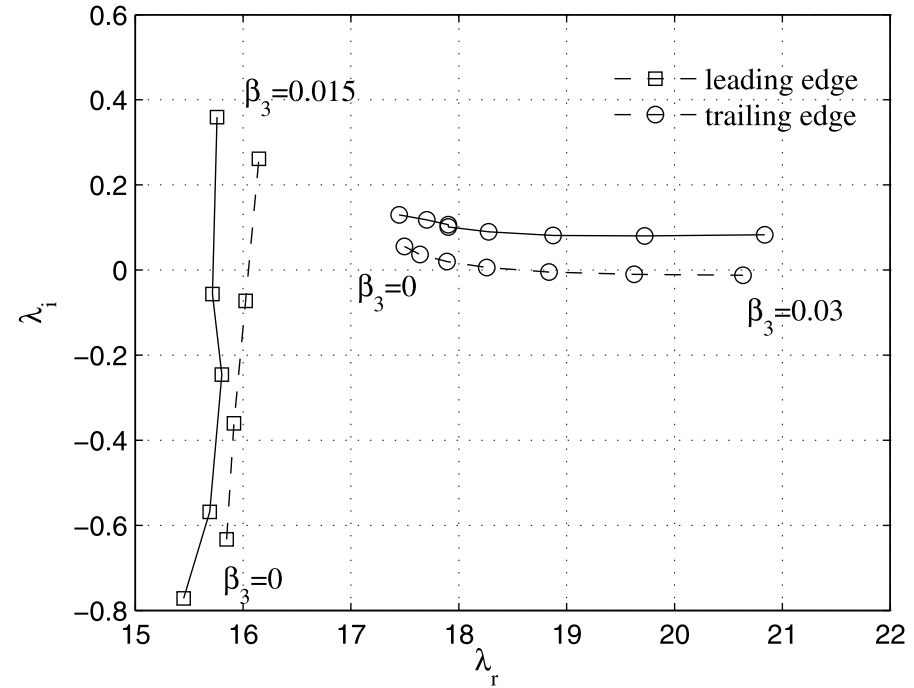

(b)

Fig. 7. Trajectories of the discrete eigenvalues as $\beta_{3}$ varies for the previous values of $\delta, \beta, \mu$ and $\nu$, and for $\epsilon=1.0$ and (a) $T_{r}=0$ and (b) $T_{r}=0.26$ (dashed lines) and $T_{r}=0.3$ (solid lines).

is present is similar to the one observed in the absence of IRS, resulting in this eigenvalue being pushed toward the $\lambda_{i}>0$ region, and thus in the elimination of explosions on the leading side of the soliton. Note that the real part of this eigenvalue does not change as much with $\beta_{3}$ as in the case of $T_{r}=0$, whereas exactly the opposite happens to the eigenvalue associated with explosions on the trailing edge. Furthermore, even though it is the real part of this eigenvalue that changes the most as $\beta_{3}$ varies, the small variation of its imaginary part can actually result in two very different situations. Effectively, while for $T_{r}=0.3$ and for the range of $\beta_{3}$ considered this imaginary part is always positive, for $T_{r}=0.26$ and $\beta_{3} \gtrsim 0.018$ this eigenvalue returns to the unstable region. Since this value of $\beta_{3}$ is larger than the one required to eliminate explosions on the leading side of the soliton $\left(\beta_{3} \gtrsim 0.011\right)$, propagation of pulses with no explosions for $T_{r}=0.26$ will be allowed, but only for a small interval of $\beta_{3}$ (roughly between 0.011 and 0.018 ). On the contrary, elimination of explosions for $T_{r}=0.3$ will be possible for a much larger interval $0.011 \lesssim \beta_{3} \lesssim 0.3$, with the lower and upper limits corresponding, respectively, to the elimination of the leading eruptions by TOD and to the transition to front kind of solutions briefly referred in Section 2.1. It is also important to point out that the combination of TOD and IRS allows stable propagation at a much lower IRS strength than with IRS alone and, moreover, at values of $T_{r}$ which are compatible with the IRS term representing an approximation to the full Raman term that affects propagation of pulses larger than 50 fs in nonlinear optical fibers [23,18].

The trajectories for the case $\epsilon=1.5$ and $T_{r}=0$ are very similar to the ones for $\epsilon=1.0$, with the two eigenvalues separating and moving into different directions as $\beta_{3}$ increases. As for $\epsilon=1.0$, this will result in the elimination of the leading edge explosions for $\beta_{3}$ positive, and of the trailing edge ones for $\beta_{3}$ negative. Thus, as already mentioned, we would expect that the combination of IRS with strengths between the two critical $T_{r} \mathrm{~s}$ and negative TOD would be able to eliminate explosions on both sides. However, when computing the trajectories of the discrete eigenvalues with those IRS strengths plus negative TOD, we found that the trajectory of the eigenvalue that was already stable in the absence of TOD was similar to the one for $T_{r}=0.26$ in Fig. $7(\mathrm{~b})$, but with this eigenvalue returning to the unstable region for a $\left|\beta_{3}\right|$ smaller than the one required to turn the other eigenvalue stable. This means that there is no regime that corresponds to both eigenvalues being stable and, thus, no stable propagation of solitons is possible in this case. A similar result was obtained in all the other numerical simulations we performed with $\epsilon=1.5$ and those $T_{r} \mathrm{~s}$.

\section{Conclusions}

We have studied the dynamics of erupting solitons under the effects of TOD and IRS for two sets of the CGLE parameters. Numerical simulations were used to investigate the evolution of these pulses, and also to obtain their stationary profiles and velocities. We have found that, in the absence of IRS, the stationary profiles and their velocities are symmetric with the sign of $\beta_{3}$. Moreover, both the pulse shape asymmetry and the absolute value of $v$ increase with increasing $\left|\beta_{3}\right|$, with the pulses exhibiting a steeper edge on the leading side in case $\beta_{3}>0$ and on the trailing side otherwise. Similarly to what happens in the absence of TOD, the effect of IRS on the steady profiles characteristics manifests itself mainly on the value of the velocity, which varies almost linearly with $T_{r}$, and has a slope that depends on $\beta_{3}$. As far as the dynamical evolution in the presence of TOD is concerned, we have found that as long as $\left|\beta_{3}\right|$ exceeds a given threshold, positive TOD is able to eliminate eruptions on the leading side of the soliton, whereas negative TOD eliminates explosions on the other side. The combined action of TOD and IRS is able to eliminate explosions on both sides of the soliton for one of the two sets of CGLE parameters considered, for a much lower value of IRS than the one required with IRS alone. In some cases, especially for small strength of IRS, the addition of TOD is responsible for the reappearance of the eruptions that were already canceled by IRS. This is the cause for not being possible to obtain steady propagation in one of the CGLE parameters set for lower IRS. We also pointed out that it is possible to find a combination of CGLE, IRS and TOD parameters that results in the stable propagation of a pulse in the CGLE erupting region with zero velocity.

\section{References}

[1] A. Mecozzi, J. Moores, H. Haus, Y. Lai, Opt. Lett. 16 (1991) 1841. 
[2] Y. Kodama, A. Hasegawa, Opt. Lett. 17 (1992) 31.

[3] L. Mollenauer, J. Gordon, S. Evangelides, Opt. Lett. 17 (1992) 1575.

[4] M. Matsumoto, H. Ikeda, T. Uda, A. Hasegawa, J. Lightwave Technol. 13 (1995) 658.

[5] H. Haus, J. Fujimoto, E. Ippen, J. Opt. Soc. Am. B 8 (1991) 2068.

[6] J. Moores, Opt. Commun. 96 (1993) 65.

[7] N. Akhmediev, V. Afanasjev, Phys. Rev. Lett. 75 (1995) 2320.

[8] N. Akhmediev, V. Afanasjev, J. Soto-Crespo, Phys. Rev. E 53 (1996) 1190.

[9] V. Afanasjev, N. Akhmediev, J. Soto-Crespo, Phys. Rev. E 53 (1996) 1931.

[10] J. Soto-Crespo, N. Akhmediev, V. Afanasjev, S. Wabnitz, Phys. Rev. E 55 (1997) 4783.

[11] N. Akhmediev, A. Ankiewicz, J. Soto-Crespo, Phys. Rev. Lett. 79 (1997) 4047.

[12] J. Soto-Crespo, N. Akhmediev, A. Ankiewicz, Phys. Rev. Lett. 85 (2000) 2937.

[13] J.M. Soto-Crespo, N. Akhmediev, K.S. Chiang, Phys. Lett. A 291 (2001) 115.

[14] S. Cundiff, J. Soto-Crespo, N. Akhmediev, Phys. Rev. Lett. 88 (2002) 73903.

[15] H. Tian, Z. Li, J. Tian, G. Zhou, J. Zi, App. Phys. B 78 (2004) 199.

[16] L. Song, L. Li, Z. Li, G. Zhou, Opt. Commun. 249 (2005) 301.

[17] S. Latas, M. Ferreira, Opt. Lett. 35 (2010) 1771.

[18] M. Facão, M.I. Carvalho, S.C. Latas, M.F. Ferreira, Phy. Lett. A 374 (2010) 4844.

[19] M. Facão, M. Carvalho, Phys. Lett. A 375 (2011) 2327.

[20] S. Latas, M. Ferreira, M. Facão, Appl. Phys. B 104 (2011) 1.

[21] N. Akhmediev, J. Soto-Crespo, Phys. Rev. E 70 (2004) 036613.

[22] D. Henry, Geometric Theory of Semilinear Parabolic Equations, 3rd edition, Lecture Notes in Mathematics, vol. 840, Springer-Verlag, Berlin, 1993.

[23] G.P. Agrawal, Nonlinear Fiber Optics, Academic Press, 2001. 